\title{
Fascin-1 is associated with recurrence in solitary fibrous tumor/hemangiopericytoma
}

\author{
YUMIKO YAMAMOTO $^{1}$, YOSHIHIRO HAYASHI ${ }^{2}$, HIDEYUKI SAKAKI ${ }^{3}$ and ICHIRO MURAKAMI Y $^{1,4}$ \\ ${ }^{1}$ Department of Diagnostic Pathology, Kochi University Hospital, Kochi University; ${ }^{2}$ Equipment of Support Planning Office, \\ Kochi University, Nankoku, Kochi 783-8505; ${ }^{3}$ Department of Nutritional Sciences for Well-being Health, \\ Kansai University of Welfare Sciences, Kashiwa, Osaka 582-0026; ${ }^{4}$ Department of Pathology, \\ School of Medicine, Kochi University, Nankoku, Kochi 783-8505, Japan
}

Received March 31, 2021; Accepted July 15, 2021

DOI: $10.3892 /$ mco.2021.2361

\begin{abstract}
Fascin-1, an actin-bundling protein, is associated with poor prognosis in patients with various types of human carcinoma. However, research is limited on the role of fascin-1 in sarcoma. Solitary fibrous tumor (SFT) and hemangiopericytoma (HPC) are rare sarcomas derived from the mesenchyme. Although the prognosis of SFT/HPC is generally favorable, fatalities are possible with repeated recurrence and distant metastasis. The current study included a total of 20 Japanese patients, who were diagnosed with SFT/HPC and underwent surgery at Kochi University Hospital from January 2000 to December 2019. The statistical relationship between recurrence and the following variables were examined: Sex, age of onset, tumor origin, tumor size, necrosis, mitosis $\geq 1 / 10$ high power field (HPF; magnification, $x 400$ ), Ki-67 >5\% and Fascin-1. A significant association was determined between recurrence and necrosis, mitosis $\geq 1 / 10 \mathrm{HPF}$ (magnification, $\mathrm{x} 400), \mathrm{Ki}-67>5 \%$, and Fascin-1 $\geq$ 'strongly positive' $(\mathrm{P}<0.05)$. The results demonstrated that Fascin-1 immunostaining may be a highly effective and useful evaluation factor for predicting poor prognosis in patients with SFT/HPC, a fatal sarcoma of humans.
\end{abstract}

\section{Introduction}

Solitary fibrous tumor (SFT) and hemangiopericytoma (HPC) are rare tumors that derive frommesenchyme.SFTandHPC were formerly considered to be different diseases, however closely related due to similarities observed in immunohistochemical positive staining in CD34, CD99, vimentin, BCL2, and

Correspondence to: Dr Yumiko Yamamoto, Department of Diagnostic Pathology, Kochi University Hospital, Kochi University, 185-1, Kohasu, Oko-cho, Nankoku, Kochi 783-8505, Japan E-mail: yumi.esther.yamamoto@gmail.com

Key words: Fascin-1, solitary fibrous tumor, hemangiopericytoma, NGFI-A-binding protein 2-signal transducer, activator of transcription 6, Ki-67, mitosis epithelial membrane antigen (1-5). This lack of specificity in SFT/HPC occasionally caused problems in differentiating them from other tumors that are immunohistologically alike them. In 2013, three groups reported that SFT and HPC have a common gene fusion between NGFI-A-binding protein 2 (NAB2) and signal transducer and activator of transcription 6 (STAT6) (1,6,7). Thereafter, STAT6, which has dual functions as a signal transducer and as transcription activator in SFT and HPC, was recognized as the highly sensitive and specific immunohistochemical marker for SFT/HPC (2-5,8-11).

Clinical progression of SFT/HPC is different in each case. For example, we reported one case where it was diagnosed accidentally and treated completely by a successful surgery, whereas in other cases relapse occurred with local recurrence and/or multiple metastases after many years of surgical treatment followed by radiotherapy and/or intensive chemotherapy $(1,3,9,10,12-18)$. Regardless of the many attempts that have been made to classify NAB2-STAT6 fusion variants to prognose clinical characteristics of SFT/HPC, any absolute fusion variant related to malignancy has not yet been detected $(11,19,20)$. However, recently classical grading of SFT/HPC with histopathological backgrounds, such as mitosis and necrosis, have been reevaluated to detect factors that may be associated with a malignant prognosis $(15,16,21)$. Furthermore, several groups reported that $\mathrm{Ki}-67$, a protein widely known to associate with a poor prognosis in various cancers $(2,22-25)$, is related to recurrence in SFT/HPC derived from the pleura and central nervous system $(2,14,15)$. We also detected a significant relationship to recurrence between necrosis, mitosis $\geq 1 / 10$ HPF (magnification, $x 400$ ), and Ki-67 $>5 \%$ in SFT/HPC regardless of its origin (26).

In this study, we added evaluation of Fascin-1 immunostaining as a potential factor that may predict recurrence of SFT/HPC. Fascin-1, an actin-bundling protein, plays an important role in the regulation of cell adhesion, migration and invasion $(27,28)$. It is known that Fascin-1 has a strong upregulation in various human carcinomas. However, regarding sarcomas, there are only a few earlier reports (29). Our study showed that Fascin-1 was strongly associated with recurrence of SFT/SFT and it suggested that Fascin-1 could be used as a predictive factor for malignancy of SFT/HPC. 


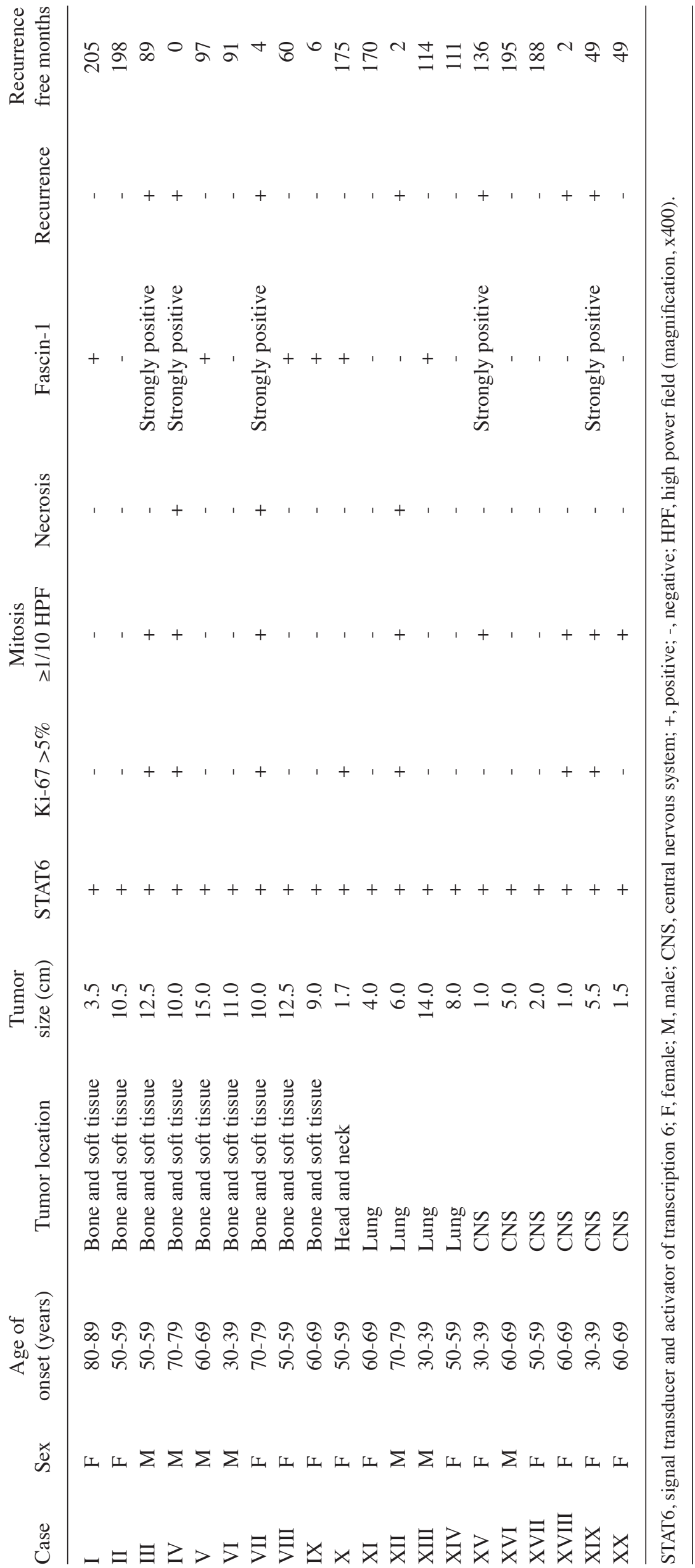


Table II. Examination of Fascin-1 staining cut off points.

\begin{tabular}{lccc}
\hline Cut-off point for Fascin-1 staining & Sensitivity & False positive rate & Concordance rate \\
\hline 'negative'-'positive', 'strongly positive' & 0.71 & 0.46 & 0.60 \\
'negative','positive'-'strongly positive' & 0.71 & 0.00 & 0.90 \\
\hline
\end{tabular}

The most effective cut-off point for Fascin-1 staining for the judgement of recurrence (+) was between 'positive' and 'strongly positive', where sensitivity, false positive rate and concordance were $0.71,0.00$ and 0.90 , respectively.

\section{Materials and methods}

Materials. A total of 20 Japanese patients, previously diagnosed with SFT/HPC at Kochi University Hospital from January 2000 to December 2019, were included in this study. Table I shows the backgrounds of these patients. All patients underwent one or more tumor resection surgery. Tissues obtained during surgery were embedded in paraffin blocks after formalin fixation and preserved. All patients were observed at Kochi University Hospital following surgery. Seven cases had one or more recurrence and two patients (cases III and VII) died due to the disease.

Immunohistochemical examination and evaluation. For the presentstudy,formalin-fixed paraffin-embedded tissue samples were freshly cut into $4 \mu \mathrm{m}$ thick slices and heat-treated with ULTRA cell conditioning 1 retrieval solution $(\mathrm{CC} 1$; Ventana Automated Systems). Immunohistochemical examination was performed using a Ventana automated system with the following antibodies: STAT6 (D-1, sc-374021, dilution 1:50; Santa Cruz Biotechnology, Inc.), Ki-67 (MIB-1, dilution 1:50; Dako; Agilent Technologies, Inc.), and anti-Fascin-1 mouse monoclonal antibody (55k-2, dilution 1:50; Dako; Agilent Technologies, Inc.). Immunohistochemical expression of STAT6, Ki-67, and Fascin-1 was evaluated in the density of the nuclear staining and graded as 'negative', 'weak', 'moderate', or 'strong'. Grades 'moderate' and 'strong', were then defined as 'positive' in terms of diagnosis. To investigate the proportion of positive Ki-67, a total of 100 tumor cells were counted at five different hot spots. Then, the mean value of positive cells was calculated and input as a percentage for statistical analysis. As for the evaluation for Fascin-1, the intensity and extent of staining were examined $(28,29)$. The intensity of staining was scored as 0 (negative), 1 (weak), 2 (moderate), or 3 (strong). The extent of staining was scored as $0(0 \%), 1(1-20 \%), 2(21-70 \%)$, and $3(71-100 \%)$. When the sum of staining intensity and extent scores was 2-4 and 5-6, it was defined as 'positive' and 'strongly positive', respectively. Independent evaluation of immunostaining was performed by two different expert pathologists who were blinded to the clinical data.

Statistical analysis. Statistical relationship was examined between recurrence and the following variables: Sex, onset age, tumor origin, tumor size, mitosis $\geq 1 / 10 \mathrm{HPF}$ (magnification, $\mathrm{x} 400$ ), necrosis, Ki-67>5\% and Fascin-1. Pearson's correlation coefficient analysis was applied to detect relationship between recurrence and sex, tumor origin, mitosis
Table III. Analyses of the relationship between recurrence and each variable.

\begin{tabular}{lc}
\hline Variables & P-value \\
\hline Male vs. female & $\mathrm{NS}$ \\
Onset age & $\mathrm{NS}$ \\
Tumor origin & $\mathrm{NS}$ \\
Tumor size (cm) & $\mathrm{NS}$ \\
Necrosis & $<0.05$ \\
Ki-67 $>5 \%$ & $<0.01$ \\
Mitosis $>1 / 10$ HPF & $<0.01$ \\
Fascin-1 $\geq$ strongly positive & $<0.01$ \\
\hline & \\
\hline & Pearson's correlation coefficient analysis was applied to detect the \\
relationship between recurrence and sex, tumor origin, necrosis, \\
mitosis $\geq 1-10$ HPF (magnification, x400), or Ki-67>5\%. Logistic \\
regression analysis was applied to compare recurrence with tumor \\
size. Wilcoxon rank test was applied to compare recurrence with \\
onset age and Fascin-1 $\geq$ 'strongly positive'.
\end{tabular}

$\geq 1 / 10 \mathrm{HPF}$ (magnification, $\mathrm{x} 400$ ), necrosis, or Ki-67>5\%. Logistic regression analysis and Wilcoxon rank test were applied to compare recurrence with tumor size and onset age, respectively. Regarding Fascin-1, after the determination of its cut-off point for recurrence as between 'positive' and 'strongly positive', Wilcoxon rank test was applied to detect the relationship between recurrence and Fascin $\geq$ 'strongly positive'. Kaplan-Meier analysis was conducted to analyze the recurrence-free survival distributions between patients with Fascin-1 'strongly positive' or not.

This study was reviewed and approved by the Ethics Committee for Clinical Research of the School of Medicine, Kochi University (ERB-105384). All procedures were carried out with the adequate understanding and written consent of each patient.

\section{Results}

Clinical background of patients. Table I includes the results of histopathological and immunochemical evaluation of all cases.

Fascin-1 staining. Table II shows sensitivity, false positive rate, and concordance rate in two proposed groups with different cut-off points of Fascin-1 immunostaining. When the cut-off 


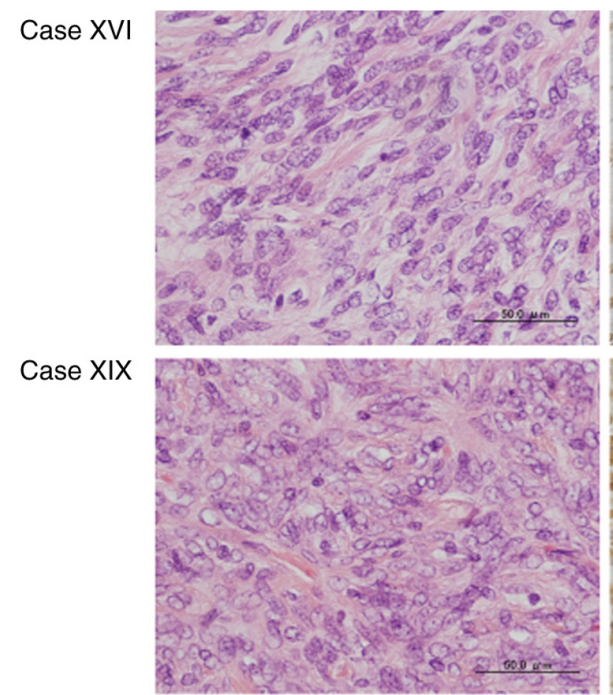

H\&E
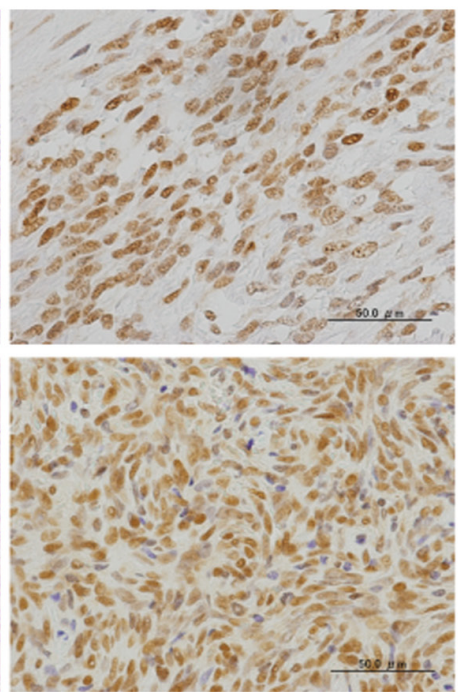

STAT6
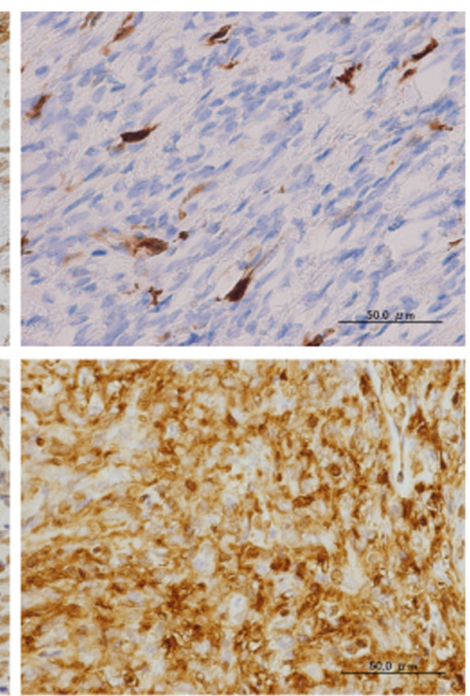

Fascin-1

Figure 1. Immunohistochemical images of H\&E, STAT6 and Fascin-1 staining in cases XVI and XIX. STAT6 was positive in each case and Fascin-1 was strongly positive in case XIX but negative in case XVI, of which staining was localized to the endothelium cells of the vessel. Scale bars, $50 \mu \mathrm{m}$. H\&E, hematoxylin and eosin; STAT6, signal transducer and activator of transcription 6.

point was set between 'positive' and 'strongly positive', the sensitivity, false positive rate, and concordance were $0.71,0.00$, and 0.90 , respectively. On the other hand, when the cut-off point was set between 'negative' and 'positive', its sensitivity, false positive rate and concordance were $0.71,0.46$ and 0.6 , respectively. By these findings, the most effective cut-off point of Fascin-1 was determined to be between 'positive' and 'strongly positive'.

Statistical analyses. Table III shows the results of relationship between recurrence and each variable. A significant relationship to reference was detected with necrosis $(\mathrm{P}<0.05)$, mitosis $\geq 1 / 10$ HPF (magnification, $\mathrm{x} 400)(\mathrm{P}<0.01), \mathrm{Ki}-67$ $>5 \%(\mathrm{P}<0.01)$, and Fascin-1 $\geq$ 'strongly positive' $(\mathrm{P}<0.01)$. Sex, onset age, tumor size, or tumor origin did not relate to recurrence. Fig. 1 shows representative images of the microscopic and immunohistochemical findings, specifically from Case XVI and Case XIX, where Fascin-1 was 'negative' in Case XVI and was 'strongly positive' in Case XIX. Fig. 2 shows Kaplan-Meier curve of patients with Fascin-1 'strongly positive' or not.

\section{Discussion}

SFT and HPC had long been regarded as different tumors since 1931, when Klemperer and Coleman first reported on primary mesenchymal tumors of the pleura (29). However, after the year 2000, controversial discussions about the classification of SFT/HPC prompted a unification of these tumors into a single disease entity. As a result, the 2013 WHO Classification of Tumors of Soft Tissue and Bone removed the term 'hemangiopericytoma' as a synonym for SFT, joining these tumors together as SFT under the category of fibroblastic/myofibroblastic tumors $(5,8,30-32)$. In the same year, the common gene fusion between NAB2 and STAT6 was discovered in SFT/HPC. The 2016 WHO Classification of Tumors of the Central Nervous System designated these tumors,

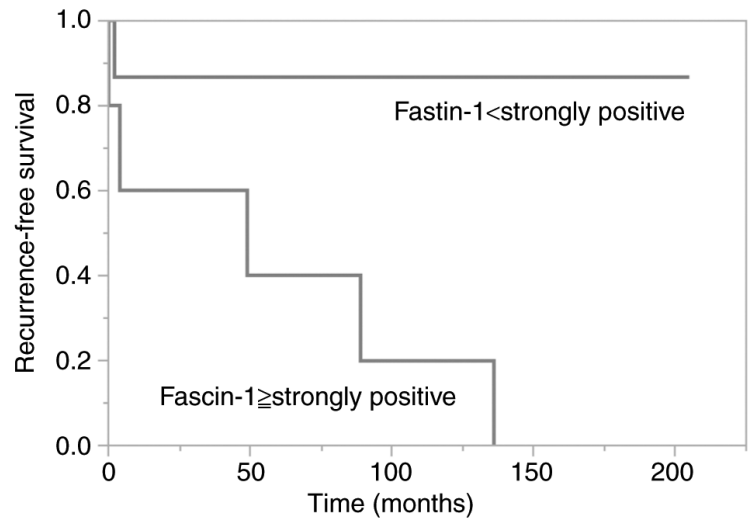

Figure 2. Kaplan-Meier curve of patients that were or were not 'strongly positive' for Fascin-1. The average observation period of patients without recurrence was 127.6 months.

characterized by the NAB2-STAT6 gene fusion, as SFT/HPC in mesenchymal/non-mesenchymal tumors $(2,9,33,34)$.

The discovery of the NAB2-STAT6 gene fusion, has resulted in both quicker and more accurate diagnosis of SFT/HPC. Cases difficult to diagnose with classical immunostainings, such as CD34, CD99, and vimentin, can easily be definitively diagnosed as SFT/HPC through the evaluation of STAT6 immunostaining. The prognosis of SFT/HPC is generally favorable, however, fatalities are possible with repeated recurrence and distant metastasis. In this study, seven of 20 cases had recurrence and two patients passed away due to the disease. The advancement of diagnostic techniques for SFT/HPC by STAT6 immunostaining may result in more cases being properly diagnosed in the future. Therefore, it is crucial for clinicians to identify patients with high risk of recurrence to adequately carry out their follow-ups.

Previously, we reported that recurrence of SFT/HPC was significantly related to necrosis, mitosis $\geq 1 / 10 \mathrm{HPF}$ (magnification, $\mathrm{x} 400$ ), and $\mathrm{Ki}-67>5 \%$ (26). Here, an 
additional factor potentially related to recurrence has been added, specifically, Fascin-1 immunostaining. Fascin-1, an actin-bundling protein, plays an important role in the regulation of cell adhesion, migration, and invasion (27-29,35). Fascin-1 widely exists in different tissues of the human body, such as mesenchyme and nervous tissue, however it is not present in most normal epithelia. Fascin-1 has been commonly observed to be highly upregulated in various human carcinomas $(27,28)$. Furthermore, the overexpression of Fascin-1 is positively correlated with poor prognosis of carcinomas, because it increases the chance of metastasis. Regarding sarcomas, few reports had been made about any relationship with Fascin-1. However, after 2019, Arlt et al reported Fascin-1 expression also correlates with progression and metastasis in osteosarcoma and chondrosarcoma (29). Additionally, Richmond et al reported that Fascin-1 is a mediator of invasion in uterine carcinosarcoma as a component of epithelial-mesenchymal transition (35).

Since 2012, pazopanib hydrochloride, a broad-spectrum tyrosine kinase inhibitor, has been approved for the treatment of soft tissue sarcoma in Japan, and its effectiveness has been reported in several papers $(36,37)$. In this study, three patients (Case VII, XV and XI) had been treated with pazopanib hydrochloride, however, Case VII patient passed away within six months following its administration. The development of new medicines that may directly target the NAB2-STAT6 gene fusion is desired for patients with SFT/HPC. Furthermore, additional therapeutics that may target and inhibit Fascin-1 will also greatly benefit patients with malignant tumors.

We attempted to detect the relationship between Fascin-1 immunostaining and recurrence of SFT/HPC. Presently, classical histological findings, such as mitosis and necrosis, are generally accepted to be useful to prognose its recurrence. As shown in Table I, sensitivity of mitosis was excellent, but there was a false-positive case. As for necrosis, its specificity was excellent, but there were four false-negative cases, which implied necrosis was not a clinically favorable factor to predict its recurrence. Regarding Fascin-1, as shown in Tables I and II, sensitivity was 0.71 and two cases had false-negative, although, its false-positive rate was 0.0 and it was negative in Case XX, where mitosis had a false-positive. By using two factors, Fascin-1 and mitosis, recurrence in SFT/HPC could be prognosed more accurately. To confirm the benefit of Fascin-1 as a factor to predict recurrence in SFT/HPC, further studies with more cases should be performed in the future. However, in this study, we observed Fascin-1 immunostaining to be one of the most effective and useful evaluation factors to predict poor prognosis in patients with SFT/HPC. Through a meticulous histological and immunochemical observation of these factors after the initial surgery, clinicians should be better informed during follow-ups with patients most at risk for recurrence and subsequently able to treat them at early stages of recurrence.

The evaluation of Fascin-1 immunostaining is useful for recurrence prediction of SFT/HPC. These data indicate that Fascin-1 may play an important role in the recurrence of SFT/HPC, one of sarcomas.

\section{Acknowledgments}

Not applicable.

\section{Funding}

No funding was received.

\section{Availability of data and materials}

The datasets used and/or analyzed during the current study are available from the corresponding author on reasonable request.

\section{Authors' contributions}

YY and YH conceived the current study. YY and YH performed the histological examination. YY and HS performed statistical analysis. YY and IM made substantial contributions to study conception and design. IM critically revised the manuscript and gave final approval for the manuscript to be published. All authors read and approved the final manuscript. YY and YH confirmed the authenticity of all the raw data.

\section{Ethics approval and consent to participate}

The current study was reviewed and approved by the Ethics Committee for Clinical Research of the School of Medicine, Kochi University (approval no. ERB-105384). All procedures were carried out with adequate understanding and the written consent of each patient.

\section{Patient consent for publication}

Not applicable.

\section{Competing interests}

The authors declare that they have no competing interests.

\section{References}

1. Robinson DR, Wu YM, Kalyana-Sundaram S, Cao X, Lonigro RJ, Sung YS, Chen CL, Zhang L, Wang R, Su F, et al: Identification of recurrent NAB2-STAT6 gene fusions in solitary fibrous tumor by integrative sequencing. Nat Genet 45: 180-185, 2013.

2. Shukla P, Gulwani HV, Kaur S and Shanmugasundaram D: Reappraisal of morphological and immunohistochemical spectrum of intracranial and spinal solitary fibrous tumors/hemangiopericytomas with impact on long-term follow-up. Indian J Cancer 55: 214-221, 2018.

3. Davanzo B, Emerson RE, Lisy M, Koniaris LG and Kays JK: Solitary fibrous tumor. Transl Gastroenterol Hepatol 3: 94, 2018.

4. Zhang Q, Qin J, Li Y and Wu T: Primary solitary fibrous tumor of kidney: A case report and literature review. Urol Case Rep 23: 92-94, 2019.

5. Doyle LA, Vivero M, Fletcher CD, Mertens F and Hornick JL: Nuclear expression of STAT6 distinguishes solitary fibrous tumor from histologic mimics. Mod Pathol 27: 390-395, 2014.

6. Chmielecki J, Crago AM, Rosenberg M, O'Connor R, Walker SR, Ambrogio L, Auclair D, McKenna A, Heinrich MC, Frank DA and Meyerson M: Whole-exome sequencing identifies a recurrent NAB2-STAT6 fusion in solitary fibrous tumors. Nat Genet 45: 131-132, 2013.

7. Mohajeri A, Tayebwa J, Collin A, Nilsson J, Magnusson L, von Steyern FV, Brosjö O, Domanski HA, Larsson O, Sciot R, et al: Comprehensive genetic analysis identifies a pathognomonic NAB2/STAT6 fusion gene, nonrandom secondary genomic imbalances, and a characteristic gene expression profile in solitary fibrous tumor. Genes Chromosomes Cancer 52: 873-886, 2013. 
8. Schweizer L, Koelsche C, Sahm F, Piro RM, Capper D Reuss DE, Pusch S, Habel A, Meyer J, Göck T, et al: Meningeal hemangiopericytoma and solitary fibrous tumors carry the NAB2-STAT6 fusion and can be diagnosed by nuclear expression of STAT6 protein. Acta Neuropathol 125: 651-658, 2013.

9. Rinaldo L, Xu SCY, Eggers SD, Salomão DR, Chen JJ and Raghunathan A: Rare occurrence of an intraocular choroida solitary fibrous tumor/hemangiopericytoma. Ocul Oncol Pathol 4: 213-219, 2018

10. Tai HC, Chuang IC, Chen TC, Li CF, Huang SC, Kao YC, Lin PC, Tsai JW, Lan J, Yu SC, et al: NAB2-STAT6 fusion types account for clinicopathological variations in solitary fibrous tumors. Mod Pathol 28: 1324-1335, 2015.

11. Kakkar A, Sakthivel P, Rajeshwari M, Kairo A and Sharma MC: Recurrent sinonasal CD34-negative malignant solitary fibrous tumor diagnosed on STAT6 immunohistochemistry and NAB2-STAT6 fusion. Head Neck Pathol 14: 250-256, 2020.

12. Penel N, Amela EY, Decanter G, Robin YM and Marec-Berard P. Solitary fibrous tumors and so-called hemangiopericytoma. Sarcoma 2012: 690251, 2012.

13. Thway K, Ng W, Noujaim J, Jones RL and Fisher C: The current status of solitary fibrous tumor: Diagnostic features, variants, and genetics. Int J Surg Pathol 24: 281-292, 2016.

14. Schmid S, Csanadi A, Kaifi JT, Kübler M, Haager B, Kayser G, Passlick B and Wiesemann S: Prognostic factors in solitary fibrous tumors of the pleura. J Surg Res 195: 580-587, 2015.

15. Diebold M, Soltermann A, Hottinger S, Haile SR, Bubendorf L, Komminoth P, Jochum W, Grobholz R, Theegarten D, Berezowska S, et al: Prognostic value of MIB-1 proliferation index in solitary fibrous tumors of the pleura implemented in a new score-a multicenter study. Respir Res 18: 210, 2017.

16. Fountas KN, KapsalakiE, Kassam M,Feltes CH,Dimopoulos VG, Robinson JS and Smith JR: Management of intracranial meningeal hemangiopericytomas: Outcome and experience. Neurosurg Rev 29: 145-153, 2006.

17. Robinson LA: Solitary fibrous tumor of the pleura. Cancer Control 13: 264-269, 2006.

18. Ghose A, Guha G, Kundu R, Tew J and Chaudhary R: CNS hemangiopericytoma: A systematic review of 523 patients. Am J Clin Oncol 40: 223-227, 2017.

19. Yuzawa S, Nishihara H, Wang L, Tsuda M, Kimura T, Tanino M and Tanaka S: Analysis of NAB2-STAT6 gene fusion in 17 cases of meningeal solitary fibrous tumor/hemangiopericytoma: Review of the literature. Am J Surg Pathol 40: 1031-1040, 2016.

20. Vogels R, Macagno N, Griewank K, Groenen P, Verdijk M, Fonville J, Kusters B; French CNS SFT/HPC Consortium; Dutch CNS SFT/HPC Consortium, Figarella-Branger D, et al: Prognostic significance of NAB2-STAT6 fusion variants and TERT promotor mutations in solitary fibrous tumors/hemangiopericytomas of the CNS: Not (yet) clear. Acta Neuropathol 137: 679-682, 2019

21. England DM, Hochholzer L and McCarthy MJ: Localized benign and malignant fibrous tumors of the pleura. A clinicopathologic review of 223 cases. Am J Surg Pathol 13: 640-658, 1989.

22. Piri R, Ghaffari A, Azami-Aghdash S, Ali-Akbar YP, Saleh P and Naghavi-Behzad M: Ki-67/MIB-1 as a prognostic marker in cervical cancer-a systematic review with meta-analysis. Asian Pac J Cancer Prev 16: 6997-7002, 2015.

23. Prueter J, Norvell D and Backous D: Ki-67 index as a predictor of vestibular schwannoma regrowth or recurrence. J Laryngol Otol 133: 205-207, 2019.

24. Kammerer-Jacquet SF, Ahmad A, Møller H, Sandu H, Scardino P, Soosay G, Beltran L, Guzick J and Berney DM: Ki-67 is an independent predictor of prostate cancer death in routine needle biopsy samples: Proving utility for routine assessments. Mod Pathol 32: 1303-1309, 2019.
25. Go SI, Ko GH, Lee WS, Lee JH, Jeong SH, Lee YJ, Hong SC and Ha WS: The use of CD44 variant 9 and Ki-67 combination can predicts prognosis better than their single use in early gastric cancer. Cancer Res Treat 51: 1411-1419, 2019.

26. Yamamoto Y, Hayashi Y and Murakami I: Recurrence of solitary fibrous tumor/hemangiopericytoma could be predicted by Ki-67 regardless of its origin. Acta Med Okayama 74: $335-343,2020$

27. Hayashi Y, Osanai M and Lee GH: Fascin-1 expression correlates with repression of E-cadherin expression in hepatocellular carcinoma cells and augments their invasiveness in combination with matrix metalloproteinases. Cancer Sci 102: 1228-1235, 2011.

28. Wang CQ, Tang CH, Chang HT, Li XN, Zhao YM, Su CM, Hu GN, Zhang T, Sun XX, Zen Y, et al: Fascin-1 as a novel diagnostic marker of triple-negative breast cancer. Cancer Med 5 : 1983-1988, 2016

29. Arlt MJ, Kuzmanov A, Snedeker JG, Fuchs B, Silvan U and Sabile AA: Fascin-1 enhances experimental osteosarcoma tumor formation and metastasis and is related to poor patient outcome. BMC Cancer 19: 83, 2019.

30. Barthelmeß S, Geddert H, Boltze C, Moskalev EA, Bieg M, Sirbu H, Brors B, Wiemann S, Hartmann A, Agaimy A and Haller F: Solitary fibrous tumors/hemangiopericytomas with different variants of the NAB2-STAT6 gene fusion are characterized by specific histomorphology and distinct clinicopathological features. Am J Pathol 184: 1209-1218, 2014.

31. Doyle LA: Sarcoma classification: An update based on the 2013 World Health Organization classification of tumors of soft tissue and bone. Cancer 120: 1763-1774, 2014

32. Jo VY and Fletcher CD: WHO classification of soft tissue tumours: An update based on the 2013 (4th) edition. Pathology 46: 95-104, 2014

33. Louis DN, Perry A, Reifenberger G, von Deimling A, Figarella-Branger D, Cavenee WK, Ohgaki H, Wiestler OD, Kleihues P and Ellison DW: The 2016 World Health Organization classification of tumors of the central nervous system: A summary. Acta Neuropathol 131: 803-820, 2016.

34. Macagno N, Vogels R, Appay R, Colin C, Mokhtari K; French CNS SFT/HPC Consortium; Dutch CNS SFT/HPC Consortium, Küsters B, Wesseling P, Figarella-Branger D, et al: Grading of meningeal solitary fibrous tumors/hemangiopericytomas: Analysis of the prognostic value of the marseille grading system in a cohort of 132 patients. Brain Pathol 29: 18-27, 2019.

35. Richmond AM, Blake EA, Torkko K, Smith EE, Spillman MA and Post MD: Fascin is associated with aggressive behavior and poor outcome in uterine carcinosarcoma. Int J Gynecol Cancer 27: 1895-1903, 2017.

36. Apra C, Alentorn A, Mokhtari K, Kalamarides M and Sanson M: Pazopanib efficacy in recurrent central nervous system hemangiopericytomas. J Neurooncol 139: 369-372, 2018.

37. Martin-Broto J, Stacchiotti S, Lopez-Pousa A, Redondo A, Bernabeu D, de Alava E, Casali PG, Italiano A, Gutierrez A, Moura DS, et al: Pazopanib for treatment of advanced malignant and dedifferentiated solitary fibrous tumour: A multicentre, single-arm, phase 2 trial. Lancet Oncol 20: 134-144, 2019.

his work is licensed under a Creative Commons Attribution-NonCommercial-NoDerivatives 4.0 International (CC BY-NC-ND 4.0) License. 